\title{
Acrylamide content in French fries prepared in households: A pilot study in Spanish homes
}

\author{
M. Mesías, C. Delgado-Andrade, F. Holgado, F.J. Morales \\ Institute of Food Science, Technology and Nutrition, ICTAN-CSIC, Madrid 28040, Spain
}

\begin{abstract}
An observational cross-sectional pilot study in 73 Spanish households was conducted to evaluate the impact of consumer practices on the formation of acrylamide during the preparation of French fries from fresh potatoes applying one stage frying. $45.2 \%$ of samples presented acrylamide concentrations above the benchmark level for French fries $(500 \mu \mathrm{g} / \mathrm{kg}) .6 .9 \%$ of samples exceeded $2000 \mu \mathrm{g} / \mathrm{kg}$ and the 95 th percentile was $2028 \mu \mathrm{g} / \mathrm{kg}$. The median and average values were significantly higher than the EFSA report for this food category, suggesting that the total exposure to acrylamide by the population could be underestimated. In this randomised scenario of cooking practices, the content of reducing sugar and asparagine did not explain the acrylamide levels. However, the chromatic parameter a* of the fried potato was a powerful tool to classify the samples according to the acrylamide benchmark level regardless of the agronomical characteristics of the potato or the consumer practices.
\end{abstract}

KEYWORDS: Acrylamide, French fries, Process contaminants, Food preparation, Consumers, Frying, Colour.

\section{INTRODUCTION}

In June 2015, the European Food Safety Agency (EFSA) concluded that the presence of acrylamide in foods potentially increases the risk of developing certain types of cancer in all age groups (EFSA, 2015). Since acrylamide is present in a wide range of foods, the exposure to this process contaminant is a public health concern and a priority for the National Food Safety Authorities. Therefore, the Directorate General for Health and Food Safety at the European Commission has established compulsory codes of practice for food business operators and benchmark levels for the reduction of acrylamide in food (European Commission, $2017)$.

French fries and sliced potato crisps are important contributors to the dietary intake of acrylamide across Europe, together with coffee and cereal-based products. The European Commission regulation identifies a benchmark level of $500 \mu \mathrm{g} / \mathrm{kg}$ for French fries (European Commission, 2017). This value is in line with the outcome of the EFSA scientific opinion on acrylamide in this food category that determines an average content of $308 \mu \mathrm{g} / \mathrm{kg}$ and $97 \mathrm{I} \mathrm{gg} / \mathrm{kg}$ at the 95th percentile (EFSA, 20I5). However, the home-cooking practices for food preparation have not been considered in the estimation of the risk.

Several variables affect acrylamide levels in potato products, such as the content of precursors in the fresh tuber, the frying conditions and the final moisture (Elmore, Koutsidis, Dodson, Mottram, \& Wedzicha, 2005). Many studies have focused on the search for strategies to mitigate the formation of acrylamide in fried potato (Palermo et al., 2016; Vinci, Mestdagh, \& De Meulenaer, 2012). However, most of them were carried out under controlled laboratory conditions with calibrated fryers, where the variety of the potato, pre-frying treatments, frying conditions and final moisture were carefully defined and controlled. Similarly, food companies have a number of mitigation strategies as compiled in the Acrylamide Toolbox (FoodDrinkEurope, 2013). The food industry is able to reproduce the processing conditions between 
batches, and supervise the critical variables with the application of procedures based on hazard analysis and critical control point principles. However, this situation cannot be extended to a private domestic setting, where the main variables of the process will differ home-to home, and time-to-time or even individual-toindividual (Langiano et al., 20I2).

Around $50 \%$ of overall acrylamide intake comes from foods cooked at home, or prepared by catering services and restaurants (Dybing et al., 2005). The lack of standardised control of frying and the different practices of food handlers seem to contribute most to the large variation and high acrylamide concentrations in French fries (Sanny, Jinap, Bakker, van Boekel, \& Luning, 20I2). In recent years, some educational initiatives link the golden colour of the fried potato to lower levels of acrylamide and, therefore, to good frying practices. In this sense, the European Association of potato processors launched the GoodFries initiative (www.goodfries.eu) for food services and consumers. The Food Standards Agency in the United Kingdom and the Spanish Food Safety Agency, launched the initiatives 'Go for Gold' (FSA, 2017) and 'golden and not toasted" (AECOSAN, 2015) respectively, helping consumers understand how to minimise acrylamide exposure at home.

Sanny, Luning, Jinap, Bakker, and van Boekel (2013) carried out an observational study in restaurants to quantify the effect of the instructions on food handlers' control decisions with respect to quality of French fries. Most frying operators exceeded the prescribed frying time, since they fried the potatoes until the colour and crispiness of French fries were consistent with their own preferences. Training of these food handlers was effective in reducing acrylamide formation. However, the information about the contribution of home-cooking practices to acrylamide exposure is very limited. The impact of different culinary procedures on acrylamide in potato-based products has been reported (Michalak, Gujska, \& Klepacka, 20II) but the contribution of the consumer practices has not been considered. Delgado-Andrade, Morales, Seiquer, and Navarro (2010) investigated the acrylamide content in several Spanish dishes. Later the same research team quantified the acrylamide content in food and dishes consumed by a Spanish group of boys aged II-14 years during a nutritional intervention period. The diet, that was balanced and free of snacks, provided a daily acrylamide intake of $0.534 \mu \mathrm{g} / \mathrm{kg}$ body weight/day (Delgado-Andrade, Mesias, Morales, Seiquer, \& Navarro, 2012). However, the menus were not home cooked, but provided by a food service catering under supervision of a chef and following standardised protocols.

Recently, Mesías, Holgado, Márquez-Ruiz, and Morales (2017) pointed out the need for additional efforts in the food distribution chain, to provide potatoes with reasonably low levels of reducing sugars, to help consumers to mitigate the formation of acrylamide in households. The situation turns more relevant in winter since only stored potato is available on the market. Cold sweetening that occurs during storage at low temperature should be avoided, to control the irreversible accumulation of reducing sugars. The effect of the seasonality of the potato has been extensively described as a factor that affects acrylamide levels (Powers, Mottram, Curtis, \& Halford, 2013). Nowadays industrial practices in the fresh potato-processing sector already consider the selection of varieties with low reducing sugar content, and a storage temperature above $6^{\circ} \mathrm{C}$ (FoodDrinkEurope, 2013).

Domestic food-preparation practices are likely to be influential in consumer exposure outcomes to acrylamide. In this respect, there is a gap in the literature regarding the expected levels of formation of acrylamide in a domestic setting. This investigation was designed based on a scenario of high variability and aimed to identify the impact of consumer practices on the acrylamide content in French fries prepared at home. 


\section{MATERIALS AND METHODS}

\section{Chemicals and reagents}

Potassium hexacyanoferrate (II) trihydrate (98\%, Carrez-I) and zinc acetate dehydrate (> 99\%, Carrez-II) were obtained from Sigma (St. Louis, MO). I3C3-labelled acrylamide ( $99 \%$ isotopic purity) was obtained from Cambridge Isotope Laboratories (Andover, MA). Formic acid (98\%), D-(+)-Glucose and methanol (99.5\%) were from Panreac (Barcelona, Spain). Deionised water was obtained from a Milli-Q Integral 5 water purification system (Millipore, Billerica, MA). All other chemicals, solvents and reagents were of analytical grade.

\section{Study design}

A cohort of seventy-five volunteers was randomly recruited from three different provinces of Spain: Badajoz (western Spain), Madrid (central Spain) and Valencia (eastern Spain). Participants were adults responsible for food preparation in their households. Volunteers were asked to prepare a batch of French fries as they normally would at home. They did not receive instructions concerning the variety and type of the potato, the preparation (peeling, cutting, soaking, blanching), the domestic frying appliance or the frying oil used. Therefore, a randomised scenario for consumer practice during the preparation of French fries was considered. Volunteers were instructed to not use deep frozen par-fried French fries, or to cut the fresh potatoes into slices for crisps. Participants were unaware of the purpose of the research and not informed in detail about the acrylamide issue, in order to avoid any influence during the experiment. They provided passive consent prior to completing the study, and did not receive any incentives for their participation. Two participants did not complete the study and their data were withdrawn; the number of households that concluded the study was 73. The pilot study was conducted from November I5 to December 2I, 2016.

\section{Sampling}

Volunteers received a 'kit of sampling' containing guidance with instructions (including photos) for sampling, and containers properly coded to collect samples. Subjects were asked to select two homogeneous fresh potatoes without external damage from the same batch. One of them was kept as it was and wrapped in aluminium foil, and the other potato was used for frying. Each participant performed the frying procedure following their usual habits, including manipulation, selection of the kitchen appliance (pan-frying or deepfrying) and type of oil as well as decision on the end-point of frying. Once cooled, the fried potato and a portion of frying oil (ca. $25 \mathrm{~mL}$ ) were collected and frozen in the containers provided. Frozen samples were placed in an airtight freezer bag and couriered in a cool bag to the laboratory together with the fresh potato. After reception, samples were photographed and stored at $-20^{\circ} \mathrm{C}$ until analysis.

\section{Questionnaire}

Volunteers were asked to fill out a questionnaire, in order to characterise their sociodemographic conditions, the type of households and the frying practices at home. In a first step, qualitative data were collected through one-to-one interviews from a sample of 17 individuals to identify specific focal points needed for the development of the questionnaire. For this first step, similar studies previously published were taken as a reference (Gatti et al., 2015; Romero, Cuesta, \& Sánchez-Muniz, 200I; Wood, Carragher, \& Davis, 2017). After that, the questionnaire was developed and validated with 35 subjects. Questions were structured in check boxes with unique or multiple possible answers. The questionnaire was divided into seven different themes, according to: i) socio-demographic information, ii) culinary habits (experience in cooking) and frequency of French fries consumption, iii) characteristics of potatoes intended for frying, iv) kitchen appliance and frying oil, v) practices related to the pre-frying stage, vi) frying stage (subjective ratio 
between the amount of potatoes respect to the dimensions of the frying utensil: less than a half, a half, more than a half or full occupancy), vii) and practices related to the after-frying stage. Participants agreed to the use of the data in the study.

\section{Characterisation of fresh potatoes}

Potatoes were weighed and characterised by length and diameter with calipers (Etalon 125, Rolle, Switzerland). A close approximation to the moisture content was determined gravimetrically after freezedrying instead of oven-drying, due to the logistical limitations of the study, in order to preserve the integrity of the tuber.

\section{Determination of reducing and total sugars}

The determination of the reducing sugar (glucose+fructose). content was performed as described by Miller (1959) with modifications from Mesías et al. (2017) as adapted to a plate-reader. Total sugars (glucose+fructose+sucrose) content were obtained after acid hydrolysis from sucrose to glucose and fructose. Results were expressed as $g$ glucose equivalents $/ \mathrm{kg}$ of fresh sample. Limit of quantification (LoQ) was set at $0.6 \mathrm{~g}$ glucose equivalents $/ \mathrm{kg}$ sample. Analysis was done in duplicate.

\section{Asparagine determination by gas chromatography-flame ionization detection (GC-FID)}

The protocol was conducted according to Farkas and Toulouee (2003) with some minor modifications. Briefly, freeze-dried fresh potato $(50 \mathrm{mg})$ was weighed into $50-\mathrm{mL}$ screw-top tubes. Then, $25 \mathrm{~mL}$ of acetonitrile $(25 \%, \mathrm{v} / \mathrm{v})$ were added into each tube stirred for $30 \mathrm{~min}$ at room temperature and then centrifuged at $10,000 \mathrm{~g}$ for $10 \mathrm{~min}$ at $4^{\circ} \mathrm{C}$. Supernatant was collected in a $50-\mathrm{mL}$ screw-top tube. Extraction was repeated with $25 \mathrm{~mL}$ of $25 \%$ acetonitrile. Supernatants were mixed and a 2 -mL aliquot was transferred into a microcentrifuge tube and frozen overnight. After defrosting and centrifugation at $5000 \mathrm{~g}$ for $5 \mathrm{~min}$ at room temperature, samples were ready for derivatization and asparagine quantification using the commercially available EZ:faast amino acid kit (Phenomenex, Torrance, CA). One hundred microlitres of the extracted samples were transferred into sample preparation vials included in the EZ:faast GC-FID free amino acid kit. The derivatisation of the potato sample extracts was performed according to the manual provided by the manufacturer and the asparagine quantified using GCFID (Agilent GC 7820A FID) equipped with an autosampler. An amino acid dedicated column (Zebron ZB-AAA capillary; $10 \mathrm{~m} \times 0.25 \mathrm{~mm}$ ) was used for the separation of amino acids. The oven was set to start at $110^{\circ} \mathrm{C}$ and increased at $32^{\circ} \mathrm{C}$ per minute up to $320^{\circ} \mathrm{C}$. An aliquot of the derivatized sample $(\mathrm{I} \mu \mathrm{L})$ was injected at $250^{\circ} \mathrm{C}$ in split mode $(15: \mathrm{I})$. The FID detector was set to $320^{\circ} \mathrm{C}$ and the carrier helium gas flow rate as kept at $1.5 \mathrm{~mL} / \mathrm{min}$ during the run External calibration was carried out with asparagine standard and results were corrected according to the recovery of norvaline, used as internal standard. Free asparagine content was expressed as $\mathrm{g} / \mathrm{kg}$ of sample (fresh matter). Analysis was done in duplicate.

\section{Determination of CIElab colour}

Measurements were made at room temperature using a HunterLab Spectrophotometer CM-3500D colorimeter (Hunter Associates, Stamford, CT). Independent measurements of a* (redness), b* (yellowness) and L* (lightness) parameters were carried out on different areas of both fresh and fried potatoes. Three and six independent measurements were performed in fresh potatoes and French fries, respectively, in order to take into account the non-homogeneous distribution of the colour within the same potato and, especially in fried potatoes, even the non- homogeneous colour between different sticks from the same tuber. These measurements provided an average value of the variability between the fried sticks. $E$ index was calculated according to the following equation: $E=\left(L^{2}+a^{2}+b^{2}\right)^{1 / 2} . \Delta a *, \Delta b *, \Delta L *$ and $\Delta E$ were calculated 
as the difference between these parameters in the fresh tuber and those in French fries, which allowed evaluation of the colour changes in the samples after frying. The equipment was calibrated with a standard calibration white plate CR-A43 (L/93.80, a/0.3I56, b/0.3319).

\section{Determination of visual colour and visual thickness}

Three untrained panellists classified the fried samples after a visual inspection with a subjective division. French fries were visually scored from the lightest to the brownest colour, and after that they were grouped as light, golden or toasted. In addition, samples were classified according to the thickness level of the fried potato, being thin ( $<5 \mathrm{~mm} ; \mathrm{n}=33$ ), medium (from 5 to $10 \mathrm{~mm} ; \mathrm{n}=28$ ), and thick (>10 mm; $\mathrm{n}=12$ ).

\section{Acrylamide determination by liquid chromatography-electrospray ionization-tandem mass spectrometry}

Acrylamide was determined in fried samples as described by Mesías and Morales (20I5). The recovery rate of acrylamide spiked in the samples was between 90 and 106\%. The relative standard deviations (RSD) for the precision, repeatability and reproducibility of the analysis were calculated as $2.8 \%, 1.2 \%$ and $2.5 \%$, respectively. The limit of the quantitation was set at $20 \mu \mathrm{g} / \mathrm{kg}$. The accuracy of the results were recently demonstrated for potato crisps in two proficiency tests launched by the Food Analysis Performance Assessment Scheme (FAPAS) program, yielding z-scores of 0.2 (Test 3065, May-June 2016) and -0.2 (Test 307I, Feb-March 2017). Results for acrylamide were expressed as $\mu \mathrm{g} / \mathrm{kg}$ of sample. Analysis was done in triplicate.

\section{Determination of polar compounds in oil}

Total polar compounds were measured in the frying oil by a handheld device Testo 270 (Testo Inc., Sparta Township, NJ) which is a rapid method based on the dielectric constant of the oil. Results were expressed as percentage of total polar material $(\mathrm{g} / \mathrm{l} 00 \mathrm{~g}$ oil). Analysis was done in triplicate.

\section{Statistical analysis}

Statistical analyses were performed using Statgraphics Centurion XV (Herndon, VA) and SPSS version 16.0 (SPSS Inc., Chicago, IL). Results were expressed as fresh weight. Raw data of acrylamide content and chromatic parameter a* (CIElab a* values) were loge-transformed to improve the statistical analyses by the approximation to normal distributions of the data. Since the chromatic component a* ranges from negative values (greenness) to positive (redness), all the values were added to 4 to make it positive. Student t-test and analysis of variance (one-way ANOVA) followed by Fisher's test were used to identify the overall significance of differences between variables. Specific significant differences between means were determined using Scheffe's multiple range test. Homogeneity of variances was determined with Levene's test. Relationships between the different variables were evaluated by computing Pearson's and Spearman's linear correlation coefficients for parametric or non-parametric variables. All statistical parameters were evaluated at $\mathrm{p}<0.05$.

\section{RESULTS AND DISCUSSION}

\section{Description of the participants and their habits of frying potatoes at home}

Seventy-three participants completed the study that included the inhome frying test and the filling of the adhoc questionnaire. The characteristics of the participants, households and frying habits were obtained from the questionnaire. Table I summarises the sociodemographic characteristics of the participants, type of households, characteristics of the fresh potato used and culinary habits for frying potatoes. The characteristic profile of the participant in this pilot-study was a Spanish woman (75\%), 36-55 years with high 
experience in cooking. The average household was constituted of at least two persons (89\%) and no individuals under the age of 18 (70\%). Most of the participants used a frying pan (80\%), olive oil $(75 \%)$, and used an amount of potatoes equivalent to half of the surface of the frying pan or the frying basket $(41 \%)$ (potato/appliance surface). Information about the potato/frying oil ratio was not reported. Volunteers washed the potatoes before frying (89\%) but only a few volunteers applied a soaking step (19\%). Most of the volunteers added salt $(84 \%)$ to the sliced potato. Participants considered the colour as the main criterion that. determines the end-point of frying (87\%). They preferred the golden colour (92\%) and potatoes crunchy on the outside and soft on the inside (85\%). The participants were asked to use only fresh potato in the pilot study in-home, but the survey showed that $77 \%$ of the participants only use fresh potatoes when cooking fried potatoes at home and $23 \%$ use both fresh and pre-fried frozen potatoes. The responders also indicated that the fresh potatoes were preferably stored indoor at room temperature $(71 \%)$ and they preferred to buy loose potatoes $(56 \%)$ in groceries or supermarkets $(88 \%)$. Although the participants did not provide the variety of the potato used in the study, it was roughly characterised by weight, length and diameter after reception. Fresh potatoes showed a mean weight of $165.9 \mathrm{~g}$ (range: 65.7-302.5 g), length of $85 \mathrm{~mm}$ (range: $52.3-131.9 \mathrm{~mm}$ ) and diameter of $62.5 \mathrm{~mm}$ (range: $42.2-76.5 \mathrm{~mm}$ ). Concerning the habits of consumption, $42 \%$ of the participants usually consumed French fries at home just once per month and nearly $30 \%$ of the volunteers once a week. It is also noteworthy to mention that nearly one third of the participants consumed this product at home exceptionally or very rarely. All the volunteers applied one stage of frying. This profile of consumer and frying practices was followed in the three regions without significant differences $(p>0.05)$.

\section{Acrylamide levels in French fries prepared in households}

Acrylamide content in the 73 samples of French fries provided by the volunteers showed a very high variability, with values ranging from 24 to $364 \mathrm{I} \mu \mathrm{g} / \mathrm{kg}$ (Table 2, Supplementary material I). The mean value was $644 \mu \mathrm{g} / \mathrm{kg}$ and the median was $443 \mu \mathrm{g} / \mathrm{kg}$. Similar levels have been reported by others in French fries (Elmore et al., 2005; Mesías et al., 2017; Sanny et al., 20I2). The proportion of samples containing acrylamide at a level above the benchmark level of $500 \mu \mathrm{g} / \mathrm{kg}$ for French fries (European Commission, 2017) was $45.2 \%$. At this point, it is noteworthy to clarify that benchmark levels are used to evaluate the performance of mitigation strategies but are not regulatory limits or safety thresholds. Moreover, a small proportion of samples (6.9\%; $\mathrm{n}=5)$ still contained levels of acrylamide exceeding $2000 \mu \mathrm{g} / \mathrm{kg}$. These data are outliers of the normal distribution of the acrylamide content.

The acrylamide content in this pilot study for French fries prepared at home was compared to the average values reported by EFSA for the food category of 'potato fried product' (EFSA, 2015). EFSA considered two datasets for the category French fries (including fresh and par-fried potato), i) data submitted by European countries in the framework of the EFSA continuous data call $(n=1378)$, and ii) data submitted by food associations collected outside official controls $(n=316)$. The results provided by the food associations for fried potato products (except potato crisps and snacks) reflect levels of acrylamide expected after the product has been prepared according to the instructions given on the package using both home and professional fryers. The median values were 196 and $170 \mu \mathrm{g} / \mathrm{kg}$, for data collected from European countries and food associations, respectively. EFSA combined both datasets, in order to perform an exposure assessment of the European population. The middle bound estimated for acrylamide were $308 \mu \mathrm{g} / \mathrm{kg}$ and $97 \mathrm{I}$ $\mu \mathrm{g} / \mathrm{kg}$ at the 95th percentile. The average acrylamide content from EFSA (308 $\mu \mathrm{g} / \mathrm{kg})$ was significantly lower than that obtained in this study $(644 \mu \mathrm{g} / \mathrm{kg})$, probably because the EFSA report did not include data of the acrylamide occurrence in households. The dataset was basically constituted by finished products sold directly by food business operators, and secondly by food services establishments. Another potential source 
of variability is that EFSA dataset did not distinguish between fresh and pre-cooked French fries as usually sold in food services and restaurants. This is a simplification of reality and the validity of the assumption depends on the kind of substance and food under investigation (Sand, Héraud, \& Arcella, 2013). In light of the results of this pilot study about in-home consumer practices, dietary acrylamide exposure of the European population would be underestimated by not considering the in-home scenario. The aim of this research was to study the expected distribution of acrylamide in a randomised scenario of consumer practices. For this reason, the exposure to acrylamide was not estimated.

\section{Factors affecting acrylamide content in French fries}

Different agronomical and processing factors are directly related to acrylamide formation in French fries (Elmore et al., 2005, 2015). The selection of varieties with low reducing sugar content, storage at controlled conditions, soaking and blanching, addition of sodium diphosphate, reduction of frying temperature, or control of the final moisture content, among other, will mitigate the formation of acrylamide (FoodDrinkEurope, 2013; Palazoğlu \& Gökmen, 2008; Pedreschi, Kaack, Granby, \& Troncoso, 2007; Sanny et al., 2012; Vinci et al., 20II). Variables causing an effect are classified into three groups; i) those characteristic of the fresh potato: reducing sugar content, total sugar content, asparagine content, colour parameters and moisture; ii) those associated with the frying process, obtained from the information provided in the questionnaire (except for the polar compounds): type of frying oil, application or not of soaking, type of domestic appliance used and content of polar compounds in the frying oil; iii) those related to the fried potatoes: colour parameters, thickness and visual colour (Supplementary material 2).

Asparagine and reducing sugars are the precursors of acrylamide, and reducing sugar is the limiting factor of acrylamide forming potential in potato products (Becalski et al., 2004; Williams, 2005). Asparagine content in potatoes depends on factors like variety, location, fertilisation, storage and processing. Asparagine content was from 0.81 to $5.82 \mathrm{~g} / \mathrm{kg}$, with a mean value of $2.65 \mathrm{~g} / \mathrm{kg}$ and a median of $2.54 \mathrm{~g} / \mathrm{kg}$ (Table 2). The difference between the minimum and the maximum values was about seven times. Higher variability was observed in the reducing sugar content (about 89 times from minimum to maximum), which varied from<LoQ to $18.77 \mathrm{~g} / \mathrm{kg}$ with an average of $4.60 \mathrm{~g} / \mathrm{kg}$ and a median of $2.86 \mathrm{~g} / \mathrm{kg}$ (Table 2). Five samples contained levels below the LoQ and were estimated as half of the LoQ for the statistical analysis. The sugar content in the tuber is determined by the genotype and pre- and post-harvest factors. In terms of molar basis, the ratio of reducing sugar to free asparagine content was 12.7-fold (from I.9 to 23.6). The acrylamide toolbox recommends a reducing sugar content lower than $4 \mathrm{~g} / \mathrm{kg}$ for fresh potatoes intended for French fries preparation. In the present study, $43.8 \%$ of the samples exhibited a content of reducing sugars higher than this recommendation. Results show that there is a large variability in the content of the precursors from tuber to tuber that explains the wide range in the levels of acrylamide. In addition, the different frying practices of the participants is another source of variability and will increase the uncertainty of the study. There is no precise information on the potato variety used, since the participants did not report it. However, it is probably not a mistake to assume that participants used stored potatoes due to the period of the year when the study was carried out and the seasonality of this food product. Similar results for asparagine and reducing sugar content in fresh tubers have been reported by other authors. Viklund, Olsson, Sjöholm, and Skog (2008) in a two-year study on five potato clones, including cold storage reported concentrations of $3.7-15.3 \mathrm{~g} / \mathrm{kg}$ and $0.9-14.9 \mathrm{~g} / \mathrm{kg}$ for asparagine and reducing sugars, respectively. Similarly, Elmore et al. (2015) studied the acrylamide precursors in twenty potato cultivars including storage conditions and reported values of $0.15-1.24 \mathrm{~g} / \mathrm{kg}$ and $0.18-21.25 \mathrm{~g} / \mathrm{kg}$ for asparagine and reducing sugars, respectively. Vivanti, Finotti, and Friedman (2006) studied acrylamide precursors in thirty-one potato 
varieties sold at retail in Italy and the United States, reporting levels from 0.15 to $7.62 \mathrm{~g} / \mathrm{kg}$ and $0.5 \mathrm{I}-12.50$ $\mathrm{g} / \mathrm{kg}$ for asparagine and reducing sugars, respectively.

The colour of French fries is one of the quality factors considered when making a decision to purchase and ultimately decide the endpoint of the frying. The colour parameters (E-index, $L^{*}, a^{*}, b^{*}$ ) in the fresh potatoes did not show great differences since the mean and median value were similar, despite the deviation (Table 2). However, the chromatic parameter $\mathrm{a}^{*}$ showed the highest variability among the fried potato samples. Moisture content in the fresh tuber was similar among the samples $(79.86 \%)$, with values ranging from $72.78 \%$ to $87.95 \%$ (Table 2). A high variability was also observed in the polar compounds content in the frying oil, which ranged between 2.50 and $27.83 \mathrm{~g} / 100 \mathrm{~g}$ oil, with an average of $6.1 \mathrm{l} \mathrm{g} / 100 \mathrm{~g}$ (Table 2). One sample exceeded the regulated maximum level of $25 \mathrm{~g} / 100 \mathrm{~g}$ oil for polar compounds. This sample corresponds to a participant that used an electric fryer without adequate supervision of the replenishment of the frying oil.

Bivariate correlations analysis was used to investigate the strength and direction of the relationship among the different quantitative parameters and the acrylamide content. Following the procedure for nonparametric variables, results were expressed as Spearman correlation coefficients (Table 3). The levels of reducing sugars content did not show a significant correlation with acrylamide (Supplementary material 3). These results were in disagreement with those reported in previous studies, where significant correlations between the sugar content in fresh potatoes and acrylamide levels after frying were demonstrated (Elmore et al., 2015; Mesías et al., 2017). The content of asparagine was also not significantly correlated to that of acrylamide. However, a strong correlation between CIElab a* values and acrylamide content was observed $(\rho=0.573, p=0.000)$. This correlation was also observed with the difference between fresh to fried product for this parameter $\left(\Delta \mathrm{a}^{*}\right)(\rho=-0.54 \mathrm{I}, \mathrm{p}=0.000)$. The acrylamide content was also correlated with CIELab $\mathrm{b}^{*}$ and $L^{*}$ parameters and, as a consequence, with E-index in fried potatoes (Mesías et al., 2017). In former studies, tubers were processed, sliced and fried under controlled conditions (i.e. Mattäus \& Haase, 20I4). Therefore, the differences were directly attributed to the characteristics of the fresh potatoes. Again, the impact of the consumer practices was not considered as a source of variability. In this randomised trial, although sugars in the fresh tuber could directly affect the acrylamide concentration in the fried samples, several factors related to consumer practices might contribute as well. It is noteworthy to mention that compared with experimental studies, Table 3 where variables are under control, randomised observational studies may not provide complete information, since it is often impossible to control all factors that affect the outcome. Consequently, the control of the extent of browning during frying is adequate but it is not enough to reduce the formation of acrylamide when a human factor is also present. Together with the golden colour of the fried potato, educational initiatives should take into account other aspects for good frying practices such as the control of the thickness, as discussed later.

The influence of the qualitative variables on the acrylamide levels in French fries was also evaluated. The acrylamide content was $\log _{\mathrm{e}}$ transformed to improve the approximation of the data to the normal distribution. The $\log _{\mathrm{e}}$ transformed acrylamide concentrations were back-transformed to non-loge acrylamide content (original scale of measurement), in order to better understand the effect of the different variables (Table 4). One-way ANOVA was carried out for the kitchen appliance, and thickness and visual colour of the fried potato. The effects of frying oil and soaking were evaluated with student $t$-analyses. Soaking, the type of appliance and thickness did not significantly affect the acrylamide content in French fries. Significant differences were found with the use of different frying oil. In a first approximation, potatoes fried in olive oil seem to form more acrylamide than those fried in sunflower oil. This outcome should be further confirmed 
since most of the volunteers (75\%, 55/73 volunteers) reported to use only olive oil as frying oil and it might not be representative. In this sense, studies including a larger number of participants and a deeper characterisation

of both the type of olive oil and number of frying cycles are necessary to confirm this effect. Literature data are not consistent on this issue and most authors report no association with oil type (Mattäus \& Haase, 2014). The stronger effect was exhibited by the visual colour of the samples. As expected, the most browned French fries (toasted) contained significantly higher levels of acrylamide than golden and light French fries $(p=0.000)$. However, the acrylamide content of light and golden French fries was not statistically different. The difference between fresh to fried potato for the CIElab $a^{*}$ values $\left(\Delta a^{*}\right)$ was not further considered, since it did not give additional strength to the analysis.

\section{Suitability of the chromatic parameter $\mathrm{a}^{*}$}

The chromatic parameter a* was able to discriminate between the three types of French fries according to the visual colour and significant differences were found when both variables were related, increasing the power of the correlation (Fig. IA). On the other hand, French fries were classified in two groups using the benchmark level of $500 \mu \mathrm{g} / \mathrm{kg}$ as the threshold: (I) samples with levels of acrylamide below the benchmark level and (2) samples with levels of acrylamide above the benchmark level. CIElab a* values were also able to satisfactorily differentiate both groups (Fig. IB). These results are relevant for household practices since the pilot study was carried out without previous selection of the raw tuber, the frying conditions nor the endpoint, which indicates a randomised scenario with a high level of uncertainty. The redness component $a^{*}$ has been previously identified as a good predictor of the formation of acrylamide in fried potato chips (Pedreschi, Moyano, Kaack, \& Granby, 2005) and potato strips (Pedreschi, Kaack, \& Granby, 2006). The reason is that this component determined by means of computer vision-based image analysis algorithms can be correlated with the acrylamide content of potato chips (Gökmen, Senyuva, Dülek, \& Çetin, 2007). Thus, computer vision-based analysis systems have been revealed as useful low-cost and non-invasive tools for the food industry to sort fried potato products according to an acrylamide threshold limit using the same algorithm (Mogol \& Gökmen, 2013; Pedreschi, Leon, Mery, \& Moyano, 2006). All the former experiments were carried out under controlled conditions (potato variety, blanching, frying oil, frying conditions, final moisture), but undercooked and overcooked potato samples were also used to build the algorithms underlying the models.

The decision of the end of the frying process is considered as the key point, since acrylamide is formed towards the end of the frying process (Palermo et al., 2016). Following the results of the present study, this end-point could be easily controlled avoiding the excessive browning of the samples and therefore the toasted colour of the French fries. These data support the need for an educational strategy for consumers promoted by different national food safety agencies, in order to link the colour of the finished processed food with the risk for high acrylamide content. However, the control of the colour is not enough to ensure the production of French fries with low acrylamide content.

Fig. 2A shows the acrylamide content in French fries regarding the visual colour. Although most of the light and golden French fries samples reported low concentrations of acrylamide, still $32.7 \%$ of the samples had levels higher than the benchmark level. Then, it should be useful to identify another variable related to acrylamide formation and that be controlled by consumers in order to reduce its levels in French fries. Samples were also classified according to the thickness level of the fried potato. Regardless of the visual colour assessment, the acrylamide content showed an inverse trend with the thickness of the fried potato (Fig. 2B), although differences were not statistically significant (Table 4). As $48.5 \%$ of the samples classified as 
thin exhibited acrylamide content higher than the indicative value, thickness should be considered as an additional factor for acrylamide mitigation in a domestic setting. This observation is in line with the FSA study (2017) that pointed out homemade potato items tend not to be finely chopped, avoiding the risk of additional acrylamide exposure through greater surface area to volume ratios. Increasing the number of participants would be necessary to assess the strength of this relationship.

\section{CONCLUSIONS}

Assessment of dietary exposure is a pivotal step in the risk assessment of acrylamide. EFSA combines specific data on consumption with occurrence data of acrylamide that have been pooled across the different European member states. However, consumer practices during the preparation of foods in-home have not been duly considered as a source of variability. This point becomes crucial for the assessment of dietary exposure of process contaminants in general and acrylamide in particular. Domestic preparation of French fries is not sufficiently controlled compared to food business operators, and it could significantly affect the global exposure to acrylamide by consumers. This randomised pilot study confirms that acrylamide content during domestic preparation of French fries differs greatly from home to home, and is nearly 2-fold the average value considered by EFSA for this food item. Hence, it is necessary to consider the frying practices in a domestic setting as a key factor to assess the real dietary acrylamide exposure of the European population. The chromatic parameter $\mathrm{a}^{*}$ was able to sort the samples with high (> $500 \mu \mathrm{g} / \mathrm{kg}$ ) and low (< $500 \mu \mathrm{g} / \mathrm{kg}$ ) acrylamide content, regardless of potato variety and storage conditions, pre-frying operations, or the frying conditions applied by the consumer. This pilot study will be extended in further research with a larger sample, including different geographical locations and culinary practices. These results will increase the understanding of the acrylamide exposure from French fries prepared and consumed in a Spanish domestic setting.

\section{CONFLICT OF INTEREST}

The authors declare that they have no conflict of interest.

\section{ACKNOWLEDGMENT}

This research is funded by the Ministry of Economy and Competitiveness (Spain) under project SAFEFRYING (AGL20I5-46234-R). The authors thank Ms. I. Alvarez, Ms. B. Díaz, Ms. S. Jiménez and Mr. MA. Martinez for their technical assistance, Dr. J. Martinez-Monzó and Dra. P. García for the validation of the questionnaire, and participants in the pilot-study.

\section{APPENDIX A. SUPPLEMENTARY DATA}

Supplementary data associated with this article can be found, in the online version, at http://dx.doi.org/10.1016/j.foodchem.2018.03.140.

\section{REFERENCES}

AECOSAN (Spanish Agency for Consumer Affairs, Food Safety and Nutrition). (2015). URL http://www.aecosan.msssi.gob.es/AECOSAN/web/noticias_y actualizaciones/temas de interes/reducir_exp osicion acrilamida.htm. Accessed 15.11.17.

Becalski, A., Lau, B. P. Y., Lewis, D., Seaman, S. W., Hayward, S., Sahagian, M., Leclerc, Y. (2004). Acrylamide in French fries: Influence of free amino acids and sugars. Journal of Agricultural and Food Chemistry, 52(I2), 380I-3806. 
Delgado-Andrade, C., Mesias, M., Morales, F. J., Seiquer, I., \& Navarro, M. P. (2012). Assessment of acrylamide intake of Spanish boys aged II-14 years consuming a traditional and balanced diet. LWT - Food Science And Technology, 46(I), 16-22.

Delgado-Andrade, C., Morales, F. J., Seiquer, I., \& Navarro, M. P. (2010). Maillard reaction products profile and intake from Spanish typical dishes. Food Research International, 43(5), I304-13 II.

Dybing, E., Farmer, P. B., Andersen, M., Fennell, T. R., Lalljle, S. P. D., Muller, D. J. G., Verger, P. (2005). Human exposure and internal dose assessments of acrylamide in food. Food and Chemical Toxicology, 43(3), 365-410.

EFSA (European Food Safety Authority) (2015). Scientific opinion on acrylamide in food. EFSA Journal, 13(6), 4104.

European Commission (2017). Comission regulation (EU) 2017/2I58 of 20 November 2017 establishing mitigation measures and benchmark levels for the reduction of the presence of acrylamide in food. Official Journal of the European Union, L304, 24-44.

Elmore, J. S., Briddon, A., Dodson, A. T., Muttucumaru, N., Halford, N. G., \& Mottram, D. S. (2015). Acrylamide in potato crisps prepared from 20 UK-grown varieties: Effects of variety and tuber storage time. Food Chemistry, 182, I-8.

Elmore, J. S., Koutsidis, G., Dodson, A. T., Mottram, D. S., \& Wedzicha, B. L. (2005). Measurement of acrylamide and its precursors in potato, wheat, and rye model systems. Journal of Agricultural and Food Chemistry, 53(4), 1286-1293.

Farkas, T., \& Toulouee, J. (2003). Asparagine analysis in food products. LC GC Europe, 2I, I4-16.

FoodDrinkEurope (2013). The Acrylamide Toolbox (pp. 57). Food Drink Europe.

FSA (Food Standards Agency). (20I7). Acrylamide in the home: Home-cooking practices and acrylamide formation. URL. https://www.food.gov.uk/science/research/chemical-safety-research/pc-research/fs 102070.

Accessed 05.12.2017.

Gatti, M. B., Cabreriso, M. S., Chain, P., Coniglio, A., Manin, M., \& Ciappini, M. C. (2015). Evaluación de la frecuencia de consumo de alimentos fritos y de las técnicas de frituras domésticas en adultos rosarinos. Invenio, 19(37), 155-165.

Gökmen, V., Senyuva, H. Z., Dülek, B., \& Çetin, A. E. (2007). Computer vision-based image analysis for the estimation of acrylamide concentrations of potato chips and french fries. Food Chemistry, I0I (2), 79I-798.

Langiano, E., Ferrara, M., Lanni, L., Viscardi, V., Abbatecola, A. M., \& De Vito, E. (2012). Food safety at home: Knowledge and practices of consumers. Zeitschrift Fur Gesundheitswissenschaften, 20(I), 47-57.

Mattäus, B., \& Haase (2014). Acrylamide. Still a matter of concern for fried potato food? European Journal of Lipid Science and Technology, II6(6), 675-687.

Mesías, M., Holgado, F., Márquez-Ruiz, G., \& Morales, F. J. (2017). Impact of the characteristics of fresh potatoes available in-retail on exposure to acrylamide: Case study for French fries. Food Control.

Mesías, M., \& Morales, F. J. (2015). Acrylamide in commercial potato crisps from Spanish market: Trends from 2004 to 2014 and assessment of the dietary exposure. Food And Chemical Toxicology, 81, 104-I I0.

Michalak, J., Gujska, E., \& Klepacka, J. (20II). The effect of domestic preparation of some potato products on acrylamide content. Plant Foods for Human Nutrition (Dordrecht, Netherlands), 66(4), 307-3। 2.

Miller, G. L. (1959). Use of dinitrosalicylic acid reagent for determination of reducing sugars. Analytical Chemistry, 31, 426-428.

Mogol, B. A., \& Gökmen, V. (20I3). Computer vision based analysis of foods - A nondestructive colour measurement tool to monitor quality and safety. Journal of the Science of Food And Agriculture n/a-n/a.

Palazoğlu, T. K., \& Gökmen, V. (2008). Reduction of acrylamide level in French fries by employing a temperature program during frying. Journal of Agricultural and Food Chemistry, 56(15), 6162-6166. 
Palermo, M., Gokmen, V., De Meulenaer, B., Ciesarova, Z., Zhang, Y., Pedreschi, F., \& Fogliano, V. (2016). Acrylamide mitigation strategies: Critical appraisal of the FoodDrinkEurope toolbox. Food \& Function, 7(6), 2516-2525.

Pedreschi, F., Kaack, K., \& Granby, K. (2006a). Acrylamide content and color development in fried potato strips. Food Research International, 39, 40-46.

Pedreschi, F., Kaack, K., Granby, K., \& Troncoso, E. (2007). Acrylamide reduction under different pretreatments in French fries. Journal of Food Engineering, 79(4), 1287-I294.

Pedreschi, F., Leon, J., Mery, D., \& Moyano, P. (2006b). Development of a computer vision system to measure the color of potato chips. Food Research International, 39(10), 1092-1098.

Pedreschi, F., Moyano, P., Kaack, K., \& Granby, K. (2005). Color changes and acrylamide formation in fried potato slices. Food Research International, 38, I-9.

Powers, S. J., Mottram, D. S., Curtis, A., \& Halford, N. G. (2013). Acrylamide concentrations in potato crisps in Europe from 2002 to 20I I. Food Additives \& Contaminants: Part A, 30(9), 1493-1500.

Romero, A., Cuesta, C., \& Sánchez-Muniz, F. J. (200I). Utilización de freidora doméstica entre universitarios madrileños. Aceptación de alimentos congelados fritos en aceite de oliva virgen extra, girasol y gisarol alto oleico. Grasas y Aceites, 52(I), 38-44.

Sand, S., Héraud, F., \& Arcella, D. (20/3). The use of chemical occurrence data at European vs. national level in dietary exposure assessments: A methodological study. Food and Chemical Toxicology, 62, 7-15.

Sanny, M., Jinap, S., Bakker, E. J., van Boekel, M. A. J. S., \& Luning, P. A. (2012). Possible causes of variation in acrylamide concentration in French fries prepared in food service establishments: An observational study. Food Chemistry, I32(I), I34-143.

Sanny, M., Luning, P. A., Jinap, S., Bakker, E. J., \& van Boekel, M. A. J. S. (2013). Effect of frying instructions for food handlers on acrylamide concentration in french fries: an explorative study. Journal of Food Protection, 76(3), 462-472.

Vinci, R., Mestdagh, F., \& De Meulenaer, B. (2012). Acrylamide formation in fried potato products - Present and future, a critical review on mitigation strategies. Food Chemistry, 133(4), I I38-I I 54.

Vinci, R., Mestdagh, F.d.r., Van Poucke, C., Kerkaert, B., de Muer, N., Denon, Q., De Meulenaer, B. (20II). Implementation of acrylamide mitigation strategies on industrial production of French fries: Challenges and pitfalls. Journal of Agricultural and Food Chemistry, 59(3), 898-906.

Viklund, G. A., Olsson, K. M., Sjöholm, I. M., \& Skog, K. I. (2008). Variety and storage conditions affect the precursor content and amount of acrylamide in potato crisps. Journal of the Science of Food and Agriculture, 88(2), 305-312.

Vivanti, V., Finotti, E., \& Friedman, M. (2006). Level of acrylamide precursors asparagine, fructose, glucose, and sucrose in potatoes sold at retail in Italy and in the United States. Journal of Food Science, 7I (2), C8IC85.

Williams, J. S. E. (2005). Influence of variety and processing conditions on acrylamide levels in fried potato crisps. Food Chemistry, 90(4), 875-88I.

Wood, K., Carragher, J., \& Davis, R. (2017). Australian consumer's insights into potatoes - Nutritional knowledge, perceptions and beliefs. Appetite, 114, 169-174. 


\section{FIGURES AND TABLES}

Table I. Characteristics of the participants, type of the households, frequency of consumption, choice for purchase, storage of the fresh potatoes and preferences for the pre-frying, frying and end of frying stages. Row describes number of cases and percentage between brackets.

\begin{tabular}{|c|c|c|c|c|c|}
\hline \multicolumn{6}{|c|}{ Socio-demographics characteristics } \\
\hline \multirow[t]{2}{*}{ Region } & \multicolumn{2}{|l|}{ Badajoz } & \multicolumn{3}{|l|}{ Valencia } \\
\hline & $28 \quad(38.4)$ & $25 \quad(34.2)$ & $20 \quad(27.4)$ & & \\
\hline \multirow[t]{2}{*}{ Gender } & male & female & & & missing \\
\hline & $17 \quad(23.3)$ & $55 \quad(75.3)$ & & & I $\quad(1.4)$ \\
\hline \multirow[t]{2}{*}{ Nationality } & Spanish & no-Spanish & & & missing \\
\hline & $70 \quad(95.9)$ & $\mathrm{I} \quad(1.4)$ & & & $2(2.7)$ \\
\hline \multirow[t]{2}{*}{ Age-group } & $(18-35)$ & $(36-55)$ & $(56-65)$ & $>65$ & missing \\
\hline & II (15.1) & $31 \quad(42.5)$ & $14 \quad(19.2)$ & $16 \quad(21.9)$ & I $(1.4)$ \\
\hline \multirow[t]{2}{*}{ Type of household } & co-living I & co-living2 & single & students & missing \\
\hline & $28 \quad(38.4)$ & $34 \quad(46.6)$ & $7^{\circ}(9.6)$ & $2 \quad(2.7)$ & $2(2.7)$ \\
\hline \multirow[t]{2}{*}{ Individuals / home } & I-individual & 2-individuals & 3-individuals & $\geq$ 4-individuals & missing \\
\hline & $7 \quad(9.6)$ & $29 \quad(39.7)$ & $14 \quad(19.2)$ & $22 \quad(30.1)$ & I (I.4) \\
\hline \multirow[t]{2}{*}{ Individuals $<18$} & yes & no & & & missing \\
\hline & $21 \quad(28.8)$ & $(69.9)$ & & & I $(1.4)$ \\
\hline \multicolumn{6}{|c|}{ Culinary habits and frequency of consumption } \\
\hline \multirow[t]{2}{*}{ Expertise } & none & low & average & high & missing \\
\hline & I (1.4) & $4 \quad(5.5)$ & $11 \quad(15.1)$ & $56 \quad(76.7)$ & I $(1.4)$ \\
\hline \multirow[t]{2}{*}{ Consumption } & weekly & monthly & exceptionally & rarely & missing \\
\hline & $21 \quad(28.8)$ & $31 \quad(42.5)$ & $18 \quad(24.7)$ & $2 \quad(2.7)$ & $1 \quad(1.4)$ \\
\hline \multicolumn{6}{|l|}{ Potato } \\
\hline \multirow[t]{2}{*}{ Type } & fresh & frozen & both & & missing \\
\hline & $64 \quad(87.7)$ & $0 \quad(0.0)$ & $7 \quad(9.6)$ & & $2(2.7)$ \\
\hline \multirow[t]{2}{*}{ Storage } & outside & inside & both & & missing \\
\hline & $14 \quad(19.2)$ & $52 \quad(7 \mid .2)$ & $6 \quad(8.2)$ & & I $\quad(1.4)$ \\
\hline \multirow[t]{2}{*}{ Container } & bagged & loose & both & & missing \\
\hline & $10(13.7)$ & $41 \quad(56.2)$ & $22 \quad(30,1)$ & & $0 \quad(0.0)$ \\
\hline \multirow[t]{2}{*}{ Place of purchase } & groceries & local markets & supermarkets & indistinctly & missing \\
\hline & $34 \quad(46.6)$ & $5 \quad(6.8)$ & $30 \quad(4 I, I)$ & $4 \quad(5.5)$ & $0 \quad(0.0)$ \\
\hline \multicolumn{6}{|c|}{ Kitchen appliance and frying oil } \\
\hline \multirow[t]{2}{*}{ Frying oil } & olive oil & sunflower oil & & & missing \\
\hline & $55 \quad(75.3)$ & $18 \quad(24.7)$ & & & $0 \quad(0.0)$ \\
\hline \multirow[t]{2}{*}{ Kitchen appliance } & frying pan & electric fryer & others & & missing \\
\hline & $59(80.8)$ & $7 \quad(9.6)$ & $7 \quad(9.6)$ & & $0 \quad(0.0)$ \\
\hline \multicolumn{6}{|l|}{ Pre-frying stage } \\
\hline \multirow{2}{*}{ Washing } & yes & no & & & missing \\
\hline & $65(89.1)$ & $(8.2)$ & & & $2(2.7)$ \\
\hline \multirow[t]{2}{*}{ Soaking } & yes & no & & & missing \\
\hline & $14 \quad(19.2)$ & $(80.8)$ & & & $0 \quad(0.0)$ \\
\hline Salt & yes & no & & & missing \\
\hline & $61 \quad(83.6)$ & $(16.4)$ & & & $0 \quad(0.0)$ \\
\hline Frying stage & & & & & \\
\hline potato/appliance surface & $<$ half & half & $>$ half & full & missing \\
\hline & $4 \quad(5.5)$ & $(4 I .1)$ & $23 \quad(31.5)$ & $(20.5)$ & I $(1.4)$ \\
\hline
\end{tabular}




\begin{tabular}{|c|c|c|c|c|}
\hline \multirow[t]{2}{*}{ End-point criteria } & colour & taste & other & missing \\
\hline & 64 (87.7) & $7 \quad(9.6)$ & $2 \quad(2.7)$ & $\begin{array}{ll}0 & (0.0)\end{array}$ \\
\hline \multirow[t]{2}{*}{ Colour preference } & light & golden & toasted & missing \\
\hline & $5 \quad(6.8)$ & $67 \quad(91.8)$ & I $\quad(1.4)$ & $\begin{array}{ll}0 & (0.0)\end{array}$ \\
\hline \multirow[t]{2}{*}{ Texture preference } & soft & crunchy & crunchy\&soft & missing \\
\hline & $7 \quad(9.6)$ & $4 \quad(5.5)$ & $62 \quad(84.9)$ & $0 \quad(0.0)$ \\
\hline
\end{tabular}


Table 2. Descriptive analysis of the numerical variables of the pilot-study for fresh potato, process and fried potato.

\begin{tabular}{|c|c|c|c|c|c|c|c|c|c|}
\hline & Mean & SD & Min & Max & QI & Median & Q3 & $\mathbf{P}_{90}$ & $\mathbf{P}_{95}$ \\
\hline \multicolumn{10}{|l|}{ Fresh potato } \\
\hline Asparagine $(\mathrm{g} / \mathrm{kg})$ & 2.65 & 0.91 & 0.81 & 5.82 & 2.06 & 2.54 & 3.04 & 3.79 & 4.00 \\
\hline Reducing sugars $(\mathrm{g} / \mathrm{kg})$ & 4.60 & 4.57 & $<$ LoQ & 18.77 & 1.09 & 2.86 & 6.78 & 10.22 & 15.19 \\
\hline Total sugars $(\mathrm{g} / \mathrm{kg})$ & 8.67 & 6.14 & 1.47 & 29.31 & 4.27 & 6.85 & 11.29 & 15.35 & 21.85 \\
\hline Moisture (\%) & 79.86 & 3.22 & 72.78 & 87.95 & 77.00 & 80.23 & 81.92 & 83.06 & 84.94 \\
\hline E & 65.76 & 2.62 & 60.10 & 72.31 & 64.14 & 65.55 & 67.26 & 69.00 & 70.33 \\
\hline$L^{*}$ & 62.84 & 2.50 & 57.22 & 70.30 & 60.98 & 62.77 & 64.43 & 66.20 & 66.96 \\
\hline$a^{*}$ & -1.73 & 0.63 & -2.91 & 0.19 & -2.12 & -1.88 & -1.56 & -0.67 & -0.54 \\
\hline$b^{*}$ & 18.94 & 3.79 & 10.92 & 27.52 & 16.17 & 18.48 & 21.52 & 24.57 & 25.86 \\
\hline \multicolumn{10}{|l|}{ Process } \\
\hline Polar compounds (g/l00 g oil) & 6.11 & 4.62 & 2.50 & 27.83 & 3.25 & 4.00 & 8.17 & 11.00 & 12.03 \\
\hline \multicolumn{10}{|l|}{ Fried potato } \\
\hline Acrylamide ( $\mu g / k g)$ & 644 & 760 & 24 & 3641 & 183 & 443 & 794 & 1226 & 2028 \\
\hline E & 66.48 & 4.47 & 54.55 & 75.77 & 63.78 & 66.27 & 69.66 & 72.59 & 73.39 \\
\hline$L^{*}$ & 62.40 & 4.16 & 50.49 & 70.30 & 60.14 & 62.73 & 65.86 & 66.83 & 68.09 \\
\hline$a^{*}$ & 0.58 & 2.78 & -3.32 & 9.76 & -1.33 & -0.10 & 1.83 & 4.40 & 5.80 \\
\hline$b^{*}$ & 22.02 & 4.77 & $|3.3|$ & 36.22 & 19.71 & 21.21 & 23.31 & 29.24 & 31.34 \\
\hline
\end{tabular}

SD: Standard deviation. Min: Minimum. Max: Maximum. QI: First quartile. Q3: Third quartile. $\mathrm{P}_{90}$ : $90^{\text {th }}$ percentile. $P_{95}: 95^{\text {th }}$ percentile. LoQ: Limit of Quantitation. 
Table 3. Bivariate correlations analyses on the quantitative variables as possible contributors to the formation of acrylamide in French fries. Spearman correlations and level of significance.

\begin{tabular}{lcl}
\hline & Rho Spearman & P \\
\hline Fresh potato & & \\
Asparagine $(\mathrm{g} / \mathrm{kg})$ & 0.152 & 0.200 \\
Reducing sugars $(\mathrm{g} / \mathrm{kg})$ & -0.112 & 0.346 \\
Total sugars $(\mathrm{g} / \mathrm{kg})$ & 0.002 & 0.988 \\
Moisture $(\%)$ & 0.059 & 0.621 \\
$\mathrm{E}$ & 0.060 & 0.613 \\
$\mathrm{~L}^{*}$ & 0.075 & 0.530 \\
$\mathrm{a}^{*}$ & 0.061 & 0.609 \\
$\mathrm{~b}^{*}$ & 0.179 & 0.129 \\
Process & & \\
Polar compounds (g/l00 g oil) & -0.326 & $0.005^{* *}$ \\
Fried potato & & \\
$\mathrm{E}$ & -0.158 & $0.18 \mathrm{I}$ \\
$\mathrm{L}^{*}$ & $-0.27 \mathrm{I}$ & $0.020^{*}$ \\
$\mathrm{a}^{*}$ & 0.573 & $0.000^{* * *}$ \\
$\mathrm{~b}^{*}$ & 0.262 & $0.025^{*}$ \\
$\Delta \mathrm{E}$ & -0.192 & 0.104 \\
$\Delta \mathrm{L}^{*}$ & 0.295 & $0.01 \mathrm{I}^{*}$ \\
$\Delta \mathrm{a}^{*}$ & -0.541 & $0.000^{* * *}$ \\
$\Delta \mathrm{b}^{*}$ & -0.055 & 0.643 \\
\hline
\end{tabular}

$\Delta E, \Delta L^{*}, \Delta a^{*}$ and $\Delta b^{*}$ are calculated as the difference between the parameters in the fresh tuber and those in French fries. Significant values are expressed as * $(p<0.05), * *(p<0.01), * * *(p<0.001)$ 
Table 4. ANOVA-one way and t-student analyses of qualitative variables as possible contributors to the formation of acrylamide in French fries.

\begin{tabular}{|c|c|c|c|c|c|c|}
\hline & \multicolumn{4}{|l|}{$\log _{e}$} & \multicolumn{2}{|c|}{ Original scale ( $\mu \mathrm{g} / \mathrm{kg})$} \\
\hline & Mean & s.e. & groups & P-value & $\begin{array}{l}\text { Mea } \\
\mathrm{n}\end{array}$ & Lower-upper limits \\
\hline \multicolumn{7}{|l|}{ Process } \\
\hline \multicolumn{7}{|l|}{ Frying oil } \\
\hline $\begin{array}{l}\text { Olive oil } \\
\text { Sunflower oil }\end{array}$ & $\begin{array}{l}6.1475 \\
5.1699\end{array}$ & $\begin{array}{l}0.1404 \\
0.2675\end{array}$ & $\begin{array}{l}\mathrm{a} \\
\mathrm{b}\end{array}$ & 0.001 & $\begin{array}{l}468 \\
175\end{array}$ & $\begin{array}{l}353-620 \\
100-309\end{array}$ \\
\hline \multicolumn{7}{|l|}{ Soaking } \\
\hline Yes & 5.8780 & 0.3240 & a & 0.918 & 357 & $177-719$ \\
\hline No & 5.9132 & 0.1474 & $\mathrm{a}$ & & 370 & $275-497$ \\
\hline \multicolumn{7}{|l|}{ Appliance } \\
\hline Frying pan & 5.9390 & 0.1411 & $\mathrm{a}$ & 0.613 & 380 & $286-504$ \\
\hline Fryer & 5.5057 & 0.5072 & $\mathrm{a}$ & & 246 & 7I - 85I \\
\hline Others & 6.0328 & 0.5592 & $\mathrm{a}$ & & 417 & $106-1638$ \\
\hline \multicolumn{7}{|l|}{ Fried potato } \\
\hline \multicolumn{7}{|l|}{ Thickness } \\
\hline Thin & 6.0944 & 0.1756 & $\mathrm{a}$ & 0.424 & 443 & $310-634$ \\
\hline Medium & 5.7898 & 0.2454 & $\mathrm{a}$ & & 327 & $|98-54|$ \\
\hline Thick & 5.6619 & 0.3149 & a & & 288 & $144-575$ \\
\hline \multicolumn{7}{|l|}{ Visual colour } \\
\hline Light & 5.2491 & 0.2562 & $\mathrm{a}$ & 0.000 & 190 & $105-344$ \\
\hline Golden & 5.5967 & 0.1586 & $\mathrm{a}$ & & 270 & $196-372$ \\
\hline Toasted & 6.8795 & 0.1928 & b & & 972 & $649-1455$ \\
\hline
\end{tabular}

Statistical different homogeneous groups are identified with letters in different columns $(p<0.05)$. s.e.: Standard error. Values of acrylamide concentration are $\log _{\mathrm{e}}$-transformed to improve the approximation of normal distributions of the data. Means of $\log _{\mathrm{e}}$ transformed acrylamide concentration and limits in the $95 \%$ confidence interval for the mean are back-transformed to an original scale. 
Figure I. Means \pm Standard deviation plot of the chromatic a* parameter for different categories of the parameter visual colour (A), and acrylamide (ACR) level according to the benchmark level (B.L.) (B). ACR < B.L.: samples with levels of acrylamide lower than $500 \mu g / k g$. ACR > B.L.: samples with levels of acrylamide higher than $500 \mu \mathrm{g} / \mathrm{kg}$.
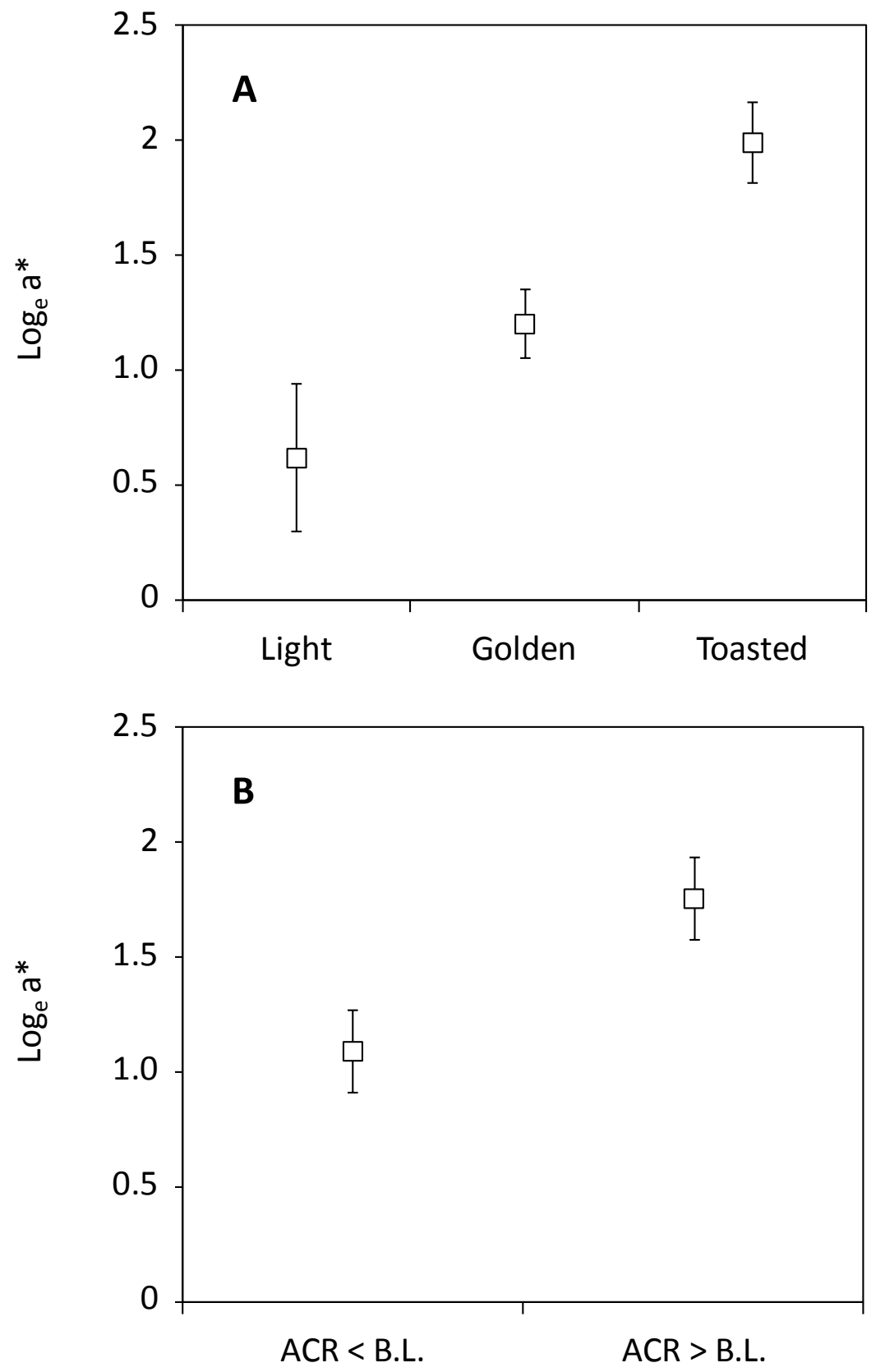
Figure 2. Box-and-whisker plot of acrylamide content ( $\mu \mathrm{g} / \mathrm{kg}$ ) according to the visual colour (light, golden, toasted) (A) and the thickness (thin, medium, thick) (B) of the French fries. ${ }^{a}$ Significant differences $(\mathrm{p}<$ 0.05).
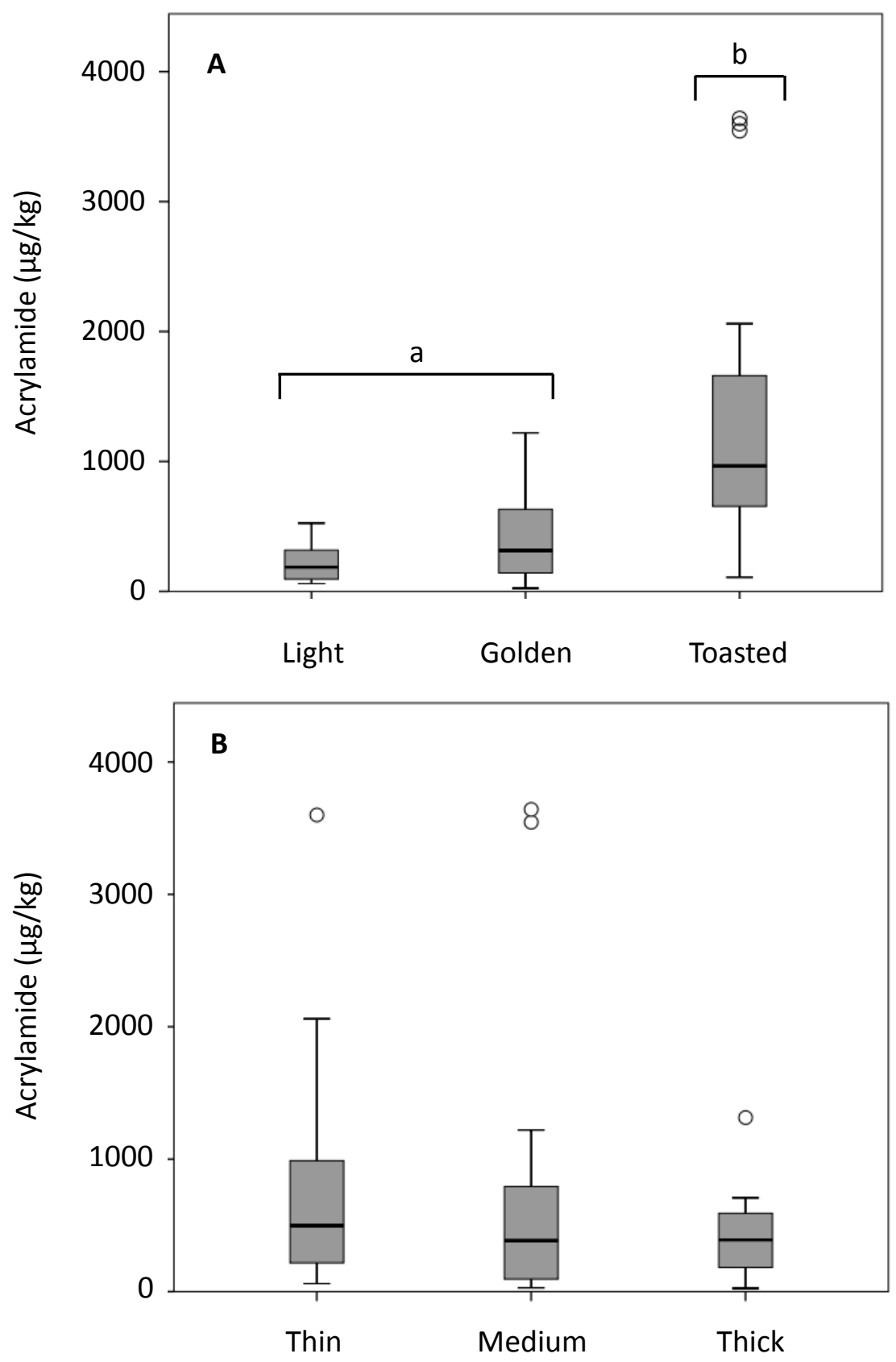